\title{
INVESTIGACIONES
}

\section{Medios de Evaluación y Desarrollo de Competencias en Educación Superior en Estudiantes de Educación Física}

\author{
Means of Assessment and Development of Competences \\ in Higher Education for Physical Education Students
}

\section{Carolina Hamodi Galán ${ }^{a}$, Juan Antonio Moreno-Murcia ${ }^{b}$, Raúl Barba Martín ${ }^{c}$}

\author{
${ }^{a}$ Universidad de Valladolid \\ Correo electrónico: carolina.hamodi@uva.es \\ ${ }^{\mathrm{b}}$ Universidad Miguel Hernández de Elche \\ Correo electrónico: j.moreno@umh.es \\ ${ }^{\mathrm{c}}$ Universidad de Valladolid \\ Correo electrónico: raulalberto.barba@uva.es
}

\begin{abstract}
RESUMEN
El objetivo del estudio es comprobar el poder de predicción de los medios de evaluación sobre las competencias en estudiantes de Educación Superior de Educación Física. Se llevaron a cabo dos estudios con muestras diferentes. Con la intención de determinar el grado de relación que podía existir entre dichas variables, se llevó a cabo un estudio descriptivo correlacional donde se midieron las competencias y los medios de evaluación. El análisis factorial exploratorio y el confirmatorio mostraron adecuadas medidas psicométricas de validez y fiabilidad de sendos instrumentos. La escala de competencias quedó compuesta por tres dimensiones (competencia genérica, competencia específica de Educación Física y competencia personal) y el instrumento de evaluación por dos dimensiones (medios de evaluación que requieren feedback y que no requieren). La principal conclusión indica que existe una relación positiva entre las competencias docentes y los medios de evaluación y además que los medios de evaluación que requieren feedback han predicho positivamente las competencias docentes en mayor medida que los medios que no lo requieren.
\end{abstract}

Palabras claves: evaluación formativa, evaluación compartida, medios de evaluación, feedback, formación inicial del profesorado.

\begin{abstract}
The objective of this study is to test the power of prediction of means of assessment for competences in universitylevel physical education students. Two studies were carried out with two different samples. A descriptive correlational study was carried out in which competences and means of assessment were measured to determine the degree of relation that might exist between these variables. Exploratory and confirmatory factor analysis showed appropriate psychometric measures of validity and reliability of both instruments. The scale of competences was composed of three dimensions (generic competence, specific competence in physical education, and personal competence) while the assessment instrument was composed of two dimensions (means of evaluation that require or do not require feedback). The leading conclusion shows a positive relation between teaching competences and means of assessment, and also demonstrates that means of assessment that required feedback positively predicted the teaching competences to a greater extent than those that did not require it.
\end{abstract}

Keywords: formative and shared assessment; means of assessment; feedback; initial teacher training. 


\section{INTRODUCCIÓN}

La Formación Inicial de Profesorado (FIP) dota a los estudiantes de competencias docentes que les permiten desarrollar su labor futura, basada en prácticas que mejoren el aprendizaje de su alumnado. Una de estas prácticas es la evaluación formativa y compartida (ÁlvarezMéndez, 2001; López-Pastor, 2006; Santos-Guerra, 1997), y en los últimos años (Arribas, Manrique \& Tabernero, 2014; Martínez, Castejón \& Santos, 2012; Ruiz-Gallardo, Ruiz \& Ureña, 2013), desde la perspectiva del alumnado, se han analizado diferentes aspectos acerca de los procesos de evaluación en esta fase. Pero son pocas las veces que se entra a analizar la perspectiva que el alumnado tiene sobre lo que ha aprendido y su utilidad para su futuro profesional. Es habitual quedarse con la percepción que los docentes tienen acerca de ello. Este proceso resulta ilógico porque limita la perspectiva del docente para conseguir una mejora en las competencias de su alumnado. Además, cada vez son más las investigaciones (Boyle \& Petriwskyj, 2014; Trede \& McEwen, 2015) que han demostrado la importancia de que exista una coherencia entre los aprendizajes que adquiere el alumnado universitario y la práctica profesional que desempeñará en el futuro. Sin embargo, no contar con la valoración del alumnado sobre este proceso puede dificultar al educador encontrar de verdad la coherencia. Aunque algunos de estos estudios hacen referencia en la toma de datos al uso de feedback en los procesos de evaluación (Ruiz-Gallardo et al., 2013) o analizan buenas prácticas que requieren feedback (Martínez-Mínguez, 2016), este aspecto no ha sido analizado en profundidad. Por lo que nuevos estudios que muestren instrumentos de evaluación formativa darán más visibilidad a estos procesos en relación al desarrollo de competencias profesionales del estudiantado.

\subsection{ENSEÑANZA POR COMPETENCIAS EN EDUCACIÓN SUPERIOR}

En España, con el objetivo de desarrollar la estructura de las enseñanzas universitarias oficiales, en 2007 se aprobó el Real Decreto1393/2007 donde se establecía la necesidad de que los estudiantes universitarios desarrollasen unas "competencias" con el objetivo de vincular la formación universitaria con el mundo profesional. Éstas son definidas como

[...] una combinación dinámica de conocimientos, habilidades, capacidades y valores. La promoción de estas competencias es el objeto del programa educativo. Las competencias cobran forma en varias unidades de curso y son evaluadas en diferentes etapas. Quien las obtiene es el estudiante. (González \& Wagenaar, 2006, p. 32).

En el Anexo I del Real Decreto citado, se dividen en "competencias generales y específicas que los estudiantes deben adquirir durante sus estudios, y que sean exigibles para otorgar el título" (RD 1393/2007, p. 23), en la misma línea que la clasificación del proyecto europeo "Tuning".

Coincidiendo con esto, en el modelo de competencias profesionales integradas (Barraza, 2007; Huerta, Pérez \& Castellanos, 2000) se especifican tres tipos de competencias: básicas o personales (son aquellas indispensables para el aprendizaje de una profesión), genéricas (son la base común de la profesión) y las específicas (son la base particular de la práctica profesional).

El proceso de enseñanza-aprendizaje centrado en el desarrollo de competencias conlleva el pasar de la tradicional transmisión de conocimientos por parte del profesor 
hacia los estudiantes, a que estos últimos sean protagonistas del aprendizaje, debiendo demostrar un "saber" (conocimientos), un "saber hacer" (habilidades) y un "ser" (actitudes) (Delgado et al., 2005). De esta forma, los estudiantes no solo deben adquirir conocimientos y contenidos, sino que también deben alcanzar ciertas capacidades durante su formación universitaria, que serán necesarias para resolver problemas profesionales, llevando a cabo un aprendizaje en contextos de la vida real (Dochy, Segers \& Dierick, 2002). Todo esto supone un necesario cambio en la metodología y, como consecuencia, también en la evaluación (Benito \& Cruz, 2005; Gessa, 2011; Semeraro, 2003; Tejada, 2011), pues ambas -metodología y evaluación- deben estar alineadas (ANECA, s.f., p. 33).

\subsection{LA EVALUACIÓN FORMATIVA Y COMPARTIDA}

La evaluación compartida es entendida como aquella que implica al alumnado en el proceso evaluativo. Por tanto, se concibe que el proceso de evaluación pertenece a todos y a todos beneficia (Santos-Guerra, 2000). En la misma línea, Brown \& Pickford (2013) la definen como el procedimiento utilizado para reconocer y responder al aprendizaje del estudiante con el fin de reforzarlo durante el propio proceso. Además, Hamodi, López y López (2017) señalan que un elemento fundamental en el proceso de evaluación formativa es la retroalimentación (feedback), mediante la cual se ofrece a los estudiantes comentarios sobre sus producciones y sugerencias sobre cómo podrían mejorar. Para el desarrollo de sistemas de evaluación formativa es fundamental escoger los medios de evaluación más apropiados, entendidos estos como "las producciones del alumnado que el profesorado puede recoger, ver y/o escuchar, y que sirven para demostrar lo que los discentes han aprendido a lo largo de un proceso determinado" (Hamodi, López \& López, 2015a, p. 155).

Para el desarrollo de sistemas de evaluación compartida se requiere la puesta en marcha de técnicas de evaluación participativas. Las técnicas son entendidas como "las estrategias que el profesorado utiliza para recoger información acerca de las producciones y evidencias creadas por el alumnado (de los medios)" (Hamodi et al., 2015a, p. 155) y aquellas que involucran al alumnado en la evaluación. Como se indicaba anteriormente, el feedback es un elemento fundamental para que la evaluación sea formativa, y por lo tanto existen medios de evaluación más afines a promover dicha retroalimentación que otros. Se podrían tener: a) medios que exigen feedback (participación del estudiante en clase o en debates, portafolios, cuadernos de campo, informes o trabajos escritos, ensayos y proyectos de aprendizaje tutorados; b) medios que no exigen feedback (exámenes de preguntas abiertas, cortas, cerradas, exámenes escritos dejando disponer de documentos y exámenes orales).

\subsection{LA EVALUACIÓN FORMATIVA Y COMPARTIDA PARA EVALUAR Y DESARROLLAR COMPETENCIAS DOCENTES}

La evaluación participativa desarrolla competencias para su futura práctica profesional (Boud \& Falchikov, 2007; Bretones, 2008; Falchikov, 2005). En este último caso es muy valioso constatar que facilita la adquisición de competencias, como por ejemplo aprender a trabajar en equipo, desarrollar la empatía o a valorar el trabajo ajeno, el aprendizaje autónomo (por ejemplo, capacidades como aprender a aprender) y el alejamiento de la memorización, desarrollando procesos cognitivos como la observación, la comparación, 
la clasificación, el análisis o la sintetización, ligados estrechamente a los procesos de formación permanente y con el aprendizaje a lo largo de la vida (Buscà et al., 2010; López, Martínez \& Julián, 2007; Muros \& Luis, 2012; Zaragoza, Luis \& Manrique, 2008), que le serán muy útiles en el mundo laboral (Ibarra, Rodríguez \& Gómez, 2012).

Además, en la Formación Inicial del Profesorado, la metodología utilizada por el docente y el sistema de evaluación asociado inciden directamente en las competencias percibidas (Hortigüela, Pérez-Pueyo \& López-Pastor, 2015). En esta etapa el alumnado desarrolla competencias y aprende mediante experiencia propia modelos de evaluación alternativos a los tradicionales (Buscà, Rivera \& Trigueros, 2012; López, 2008, 2011; López Martínez \& Julián, 2007; Martínez \& Ureña, 2008; Vallés, Ureña \& Ruiz, 2011) y pone en práctica una competencia que requerirá en su profesión: el uso de sistemas de evaluación formativa y compartida en su práctica docente (Hamodi et al., 2017; López, Manrique \& Vallés, 2011; Palacios \& López, 2013; Lorente \& Kirk, 2013).

\subsection{INSTRUMENTOS PARA MEDIR LA UTILIZACIÓN DE SISTEMAS DE EVALUACIÓN FORMATIVA Y COMPARTIDA Y DESARROLLO DE COMPETENCIAS DOCENTES}

Las escalas administradas a los estudiantes han sido los instrumentos más utilizados para evaluar las competencias docentes (Escudero, 1999; Muñoz, Ríos \& Abalde, 2002). Estas escalas han servido tanto para medir la competencia docente del profesorado, como su adquisición por parte del alumnado. Sin embargo, en los últimos años se ha buscado que los instrumentos para medir las competencias docentes tengan un carácter más comprensivo. Por ello, numerosas investigaciones han recurrido a la utilización de procesos e instrumentos diversos para medir la competencia en profesores y estudiantes (Escudero, 1999; Gargallo et al., 2010; Ivars, Fernández \& Llinares, 2016). Muñoz et al. (2002) diseñaron un instrumento compuesto por diez dimensiones (cumplimiento con las obligaciones, infraestructura, programa, conocimiento-interrelación con la materia, metodología, materiales, actitud del docente, evaluación, prácticas y satisfacción) que unían la evaluación del profesorado con aspectos comunes en la evaluación de los planes de calidad de las universidades. En los instrumentos para analizar la competencia docente adquirida por el alumnado, Gallego y Rodríguez (2014) diseñaron un cuestionario ad hoc con el que medir la competencia comunicativa del alumnado de Educación Física para ser futuros docentes. Realizaron este cuestionario en base a cinco bloques que un docente debe poseer (ser buen emisor, ser buen receptor, habilidades comunicativas en clase, habilidades con padres y colegas y habilidades en la función tutorial). Asimismo, Moreno-Murcia, Silveira y Belando (2015) diseñaron y validaron un instrumento de medida compuesto por tres dimensiones (planificación, desarrollo y resultado). Ivars, Fernández y Llinares (2016) realizaron un análisis profundo de las narrativas del alumnado, fijándose en la profundidad de sus reflexiones y sus actuaciones en torno a la competencia matemática.

Respecto al análisis específico de las técnicas y sistemas de evaluación, existen múltiples investigaciones acerca de las percepciones del profesorado, estudiantado y egresados. Por ejemplo, se ha demostrado que existe una disparidad en la percepción que, sobre los sistemas de evaluación, tienen estudiantes y profesorado (Gutiérrez et al., 2011; Martínez, Castejón \& Santos, 2012; Martínez, Santos \& Castejón, 2017) y egresados (Gutiérrez, Pérez \& Pérez, 2013; Hamodi, López \& López, 2015b), aunque las de estos últimos se encuentran próximas a las de los estudiantes. Es por todas estas razones que 
se hace necesario seguir investigando esta línea y creando instrumentos que permitan acercarse a comprender mejor estas diferencias.

El objetivo de esta investigación ha sido diseñar y validar un instrumento para medir la percepción de competencia docente y otro instrumento para medir la percepción sobre las técnicas evaluativas en estudiantes de Educación Superior de Educación Física. Partiendo de los estudios previos descritos, se espera una relación positiva entre el desarrollo de competencias docentes y los medios de evaluación utilizados; y, además, se espera que los medios de evaluación que conllevan un feedback predigan positivamente el desarrollo de competencias docentes en mayor medida que las técnicas no participativas.

\section{MÉTODO}

Se realizaron dos estudios. A continuación, se detallan los participantes, las medidas, el procedimiento y el análisis de los datos de cada uno de ellos.

\section{Estudio 1}

\section{Participantes}

La muestra estuvo compuesta por 528 estudiantes de Educación Superior ( 251 chicos y 267 chicas) de $4^{\circ}$ curso en los grados de Primaria y CAFYD, pertenecientes a seis universidades españolas, con una edad media de 22.15 años $(D T=.76)$.

Medidas

a- Competencia docente. Se diseñó la escala denominada "Percepción de la Competencia Docente en Educación Física (PCDEF)", que finalmente quedó compuesta por 24 ítems que a través de tres factores miden la percepción de competencia docente desarrollada por los estudiantes por medio de materias relacionadas con la Educación Física. Las dimensiones fueron (1) competencias genéricas, compuesta por ocho ítems (e. g., "Implicar al alumnado en su aprendizaje y en la vida del centro"); (2) competencia específica de Educación Física, compuesto por nueve ítems (e. g., "Diseñar, modificar y/o adaptar al contexto educativo situaciones motrices orientadas al desarrollo y perfeccionamiento de las habilidades motrices"); (3) competencias personales, compuesta por siete ítems (e. g., "Aprendizaje autónomo"). Estaban precedidos por la sentencia previa "Teniendo en cuenta todas las asignaturas cursadas en la carrera, ¿en qué grado crees que te han ayudado a desarrollar las siguientes competencias docentes?". Se midió a través de una escala tipo Likert que va desde 0 (nada) a 4 (muchísimo).

b- Evaluación. Se diseñó la "Escala de Medición de los Instrumentos y Procedimientos de Evaluación (EMIPE)", que finalmente quedó compuesta por 12 ítems que miden, a través de dos dimensiones, la percepción de los estudiantes de la utilización de los medios de evaluación que el profesorado utiliza para recoger información acerca de las producciones y evidencias creadas por el alumnado. Éstos han sido clasificados en dos tipos: medios que requieren feedbak, compuesta por siete ítems (e. g., "portafolios" o "proyectos de Aprendizaje tutorados") y medios que no requieren feedback, compuesta por cinco ítems (e. g., "Exámenes orales" o "exámenes de preguntas abiertas"). Estaban precedidos por la sentencia previa "¿Con cuánta frecuencia tus profesores han utilizado los siguientes instrumentos y procedimientos de evaluación en las asignaturas que has cursado?". Se 
midió a través de una escala tipo Likert cuyas opciones eran 0 (nada) a 4 (mucho). Procedimiento

La construcción del cuestionario se realizó a partir de una revisión bibliográfica donde se fueron construyendo los aspectos más importantes sobre los que debían girar los ítems de las dos escalas. La elaboración de los ítems se centró en determinar, por un lado, las competencias docentes del estudiante y, por otro, los medios de evaluación del aprendizaje. La redacción de los mismos surge de esa revisión, para posteriormente someter la batería de ítems creada a una evaluación por parte de nueve expertos en la temática, seleccionando los ítems más pertinentes por su relevancia y claridad. Respecto a la escala de la medición de las competencias docentes, ésta quedó formada por 46 ítems divididos en tres grandes campos: (a) competencias docentes de Educación Física; (b) competencias personales; y (c) competencias docentes genéricas. En cuanto a la escala de evaluación, quedó compuesta por 14 ítems, estructurada en dos dimensiones: medios de evaluación que exigen feedback y medios de evaluación que no exigen feedback. Seguidamente, se administró la batería completa a un reducido grupo de estudiantes para verificar su correcta comprensión. La impresión de los estudiantes fue que las escalas no presentaban ningún problema de comprensión, por lo que no se realizaron cambios.

Para que los estudiantes pudieran rellenar los cuestionarios en su tiempo de clase, se solicitó el permiso oportuno a los responsables de grado y docentes de la materia. Se insistió en el anonimato de las respuestas para que contestaran con honestidad y sinceridad. El tiempo requerido para su cumplimentación fue de 15 minutos, aproximadamente.

\section{Análisis de datos}

Se analizaron las propiedades psicométricas de las escalas de medida a través de un análisis factorial exploratorio de componentes principales con rotación varimax u oblimin directo con una saturación igual o mayor que .40. Se analizó la consistencia interna de cada factor mediante el coeficiente de Alfa de Cronbach. Los datos fueron analizados mediante el paquete estadístico SPSS 21.0.

\section{Estudio 2}

\section{Participantes}

La muestra estuvo compuesta por 718 estudiantes de Educación Superior (450 chicos y 268 chicas) de $4^{\circ}$ curso en los grados de Primaria $(n=341)$ y CAFYD $(n=377)$, pertenecientes a 15 Universidades españolas, con una edad media de 22.75 años $(D T=.94)$. Medidas

a- Competencia docente. Se utilizó la escala descrita en el estudio 1. La consistencia interna fue de $.86, .86$ y .84 , respectivamente.

b- Evaluación. Se utilizó la escala descrita en el estudio 1. La consistencia interna obtuvo un valor de .72 y .69 , respectivamente.

Procedimiento

Se siguió el mismo procedimiento recogido en el estudio 1.

Análisis de datos

Se calcularon los estadísticos descriptivos de todos los ítems objeto de estudio (medias y desviaciones típicas), se analizó la consistencia interna de cada factor mediante el coeficiente de Alfa de Cronbach y las correlaciones bivariadas de todas las variables. La estructura de la escala se confirmó a través de un análisis factorial confirmatorio. La 
validez del modelo de medición fue considerada a través de una serie de coeficientes fit, también llamados índices de bondad de ajuste: $\chi^{2}, \chi^{2} /$ d.f., RMSEA, RSMR y los índices incrementales (CFI, IFI y TLI). Asimismo, se comprobó el poder predictivo de las competencias docentes sobre los medios de evaluación, a través de un análisis de regresión lineal múltiple por pasos. Para examinar las posibles diferencias por sexo y tipo de estudios respecto a la percepción de competencia docente y medios de evaluación, se realizó un análisis de varianza multivariado (MANOVA). Los datos fueron analizados mediante el paquete estadístico SPSS 21.0 y AMOS 21.0.

\section{RESULTADOS}

A continuación, se presentan los resultados de cada uno de los dos estudios llevados a cabo.

\section{Estudio 1}

Análisis factorial exploratorio de la PCDEF. Se analizaron las propiedades psicométricas de la PCDEF a través de un análisis factorial exploratorio de componentes principales con rotación oblicua de oblimin, pues, según el diseño de la estructura de la misma, los factores presentaban relación. Los resultados indicaron que los ítems se agrupaban en tres factores. Después del análisis, se verificó que con la eliminación de 22 ítems que no alcanzaban el nivel mínimo de saturación (.40) se aumentaba la consistencia interna. Así, se excluyeron del instrumento los ítems y se realizó un nuevo análisis factorial sin estos ítems (Tabla 1).

Así, 24 ítems resultaron del análisis quedando agrupados en tres factores (competencias genéricas, competencias específicas de Educación Física y competencias personales) cada uno de ellos formados por ocho, nueve y siete ítems respectivamente, con autovalores mayores de 1.00 (9.48, 2.26 y 1.54), explicando una varianza total del $55.40 \%(29.55 \%$, $14.43 \%$ y $11.43 \%$, respectivamente). Los pesos de saturación oscilaron de .41 a .82. La consistencia interna de las escalas fue de $.89, .87$ y .87 , respectivamente.

Análisis factorial exploratorio de la EMIPE. Se analizaron las propiedades psicométricas de la EMIPE a través de un análisis factorial exploratorio de componentes principales con rotación varimax. Después del análisis se comprobó que dos ítems no saturaban lo suficiente. Así, se excluyó del instrumento el ítem número 3 ("Examen tipo test") y el 9 ("Pruebas prácticas de carácter físico: ejercicios físicos, situaciones de juego, etc.") y se realizó un nuevo análisis factorial de componentes principales con rotación varimax sin estos ítems (Tabla 2).

Así, 12 ítems resultaron del análisis quedando agrupados en dos factores (Medios que exigen feedback y medios que no exigen feedback) cada uno de ellos formados por siete y cinco ítems respectivamente, con autovalores mayores de 1.00 (3.68 y 1.41), explicando una varianza total del $42.48 \%$ ( $30.72 \%$ y $11.75 \%$, respectivamente). Los pesos de saturación oscilaron de .41 a .71. La consistencia interna de la escala fue de .75 y .70, respectivamente.

\section{Estudio 2}

Análisis factorial confirmatorio (CFA) de la PCDEF. Con los 24 ítems de la escala se llevó a cabo un análisis factorial confirmatorio con la misma estructura del estudio 1 de 
Estudios Pedagógicos XLIV, N$^{\circ}$ 2: 241-257, 2018

MEDIOS DE EVALUACIÓN Y DESARROLLO DE COMPETENCIAS EN EDUCACIÓN SUPERIOR EN ESTUDIANTES DE EDUCACIÓN FÍSICA

Tabla 1. Ítems, Saturación y \% Varianza del Análisis Factorial Exploratorio de la escala PCDEF

\begin{tabular}{|c|c|c|c|}
\hline & Factor 1 & Factor 2 & Factor 3 \\
\hline $\begin{array}{l}\text { 3. Elaborar y poner en práctica estrategias de atención a la } \\
\text { diversidad }\end{array}$ & .456 & & \\
\hline 4. Implicar al alumnado en su aprendizaje y en la vida del centro & .412 & & \\
\hline 5. Trabajar en equipo con otros docentes & .434 & & \\
\hline 6. Participar en la gestión del Centro & .815 & & \\
\hline 7. Informar e implicar a las familias & .820 & & \\
\hline 8. Utilizar las tecnologías de la información y la comunicación & .688 & & \\
\hline 9. Afrontar los deberes y los dilemas éticos de la profesión & .617 & & \\
\hline 10. Organizar la propia formación continua & .489 & & \\
\hline $\begin{array}{l}\text { 1. Diseñar, aplicar y analizar intervenciones didácticas en la } \\
\text { asignatura de Educación Física }\end{array}$ & & .724 & \\
\hline $\begin{array}{l}\text { 3. Diseñar, desarrollar y evaluar los procesos de enseñanza- } \\
\text { aprendizaje relativos a la actividad física y el deporte con atención } \\
\text { a las características individuales y contextuales de las personas }\end{array}$ & & .730 & \\
\hline $\begin{array}{l}\text { 6. Conocer las capacidades físicas y los factores que determinan } \\
\text { su evolución y saber aplicar sus fundamentos técnicos específicos }\end{array}$ & & 615 & \\
\hline $\begin{array}{l}\text { 12. Saber utilizar el juego como recurso didáctico y como } \\
\text { contenido de enseñanza }\end{array}$ & & 616 & \\
\hline $\begin{array}{l}\text { 14. Saber utilizar instrumentos de evaluación en la asignatura de } \\
\text { Educación Física }\end{array}$ & & .665 & \\
\hline $\begin{array}{l}\text { 17.Tener capacidad de reflexión sobre el proceso de enseñanza- } \\
\text { aprendizaje, los diferentes tipos organizativos y las distintas } \\
\text { metodologías dentro de las clases de Educación Física }\end{array}$ & & .698 & \\
\hline $\begin{array}{l}\text { 18. Conocer y comprender los procesos evolutivos corporales y } \\
\text { motrices }\end{array}$ & & .632 & \\
\hline $\begin{array}{l}\text { 19. Diseñar, modificar y/o adaptar al contexto educativo } \\
\text { situaciones motrices orientadas al desarrollo y perfeccionamiento } \\
\text { de las habilidades motrices }\end{array}$ & & .744 & \\
\hline $\begin{array}{l}\text { 20. Diseñar, desarrollar y evaluar procesos de enseñanza- } \\
\text { aprendizaje relativos a competencia motriz, con atención a las } \\
\text { características individuales y contextuales de las personas }\end{array}$ & & .709 & \\
\hline 30. Trabajo en equipo & & & -.783 \\
\hline 31. Habilidades en las relaciones interpersonales & & & -.733 \\
\hline 32. Razonamiento crítico & & & -.806 \\
\hline 33. Compromiso ético & & & -.634 \\
\hline 34. Aprendizaje autónomo & & & -.723 \\
\hline 35. Adaptación a situaciones nuevas & & & -.663 \\
\hline 36. Creatividad & & & -.745 \\
\hline$\%$ varianza & $29.55 \%$ & $14.43 \%$ & $11.43 \%$ \\
\hline$\%$ varianza total & $55.40 \%$ & & \\
\hline
\end{tabular}


Tabla 2. Ítems, Saturación y \% Varianza del Análisis Factorial Exploratorio de la escala EMIPE

\begin{tabular}{|l|c|c|}
\hline & Factor 1 & Factor 2 \\
\hline 1. Observación del profesor en clase (fichas de observación) & .492 & \\
\hline 2. El control de la participación en el aula (en grupos y debates) & .589 & \\
\hline 10. Portafolios & .656 & \\
\hline 11. Cuadernos de campo (o fichas de sesiones prácticas) & .663 & \\
\hline 12. Informes o trabajos escritos & .538 & \\
\hline 13. Ensayos a partir de textos escritos o materiales audiovisuales & .703 & \\
\hline 14. Proyectos de aprendizaje tutorados & .718 & \\
\hline 4. Examen de preguntas abiertas & & .529 \\
\hline 5. Examen de preguntas cortas (explicaciones breves) & & .702 \\
\hline 6. Examen de preguntas cerradas (definiciones) & & .690 \\
\hline 7. Exámenes escritos dejando disponer de documentos & \multicolumn{2}{|c|}{.626} \\
\hline 8. Exámenes orales & \multicolumn{2}{|c|}{$42.48 \%$} \\
\hline & $\%$ varianza total & \\
\hline
\end{tabular}

las tres dimensiones. Se comprobó que los índices obtenidos no eran adecuados: $\chi^{2}$ (75, $N=718)=1009.38, p<.000 ; \chi^{2} /$ d.f. $=4.05 ; \mathrm{CFI}=.89 ; \mathrm{IFI}=.88 ; \mathrm{TLI}=.89 ; \mathrm{RSMR}=.05 ;$ RMSEA $=.07$. Para corregir este desajuste se tuvo en cuenta las sugerencias mostradas por los índices de modificación y se correlacionaron los errores del ítem 1 con el ítem 3 y el ítem 12 con el ítem 14 de la dimensión de competencias específicas de Educación Física. Se realizó un nuevo CFA y se utilizó el método de estimación de máxima verosimilitud junto con el procedimiento de bootstrapping, ya que el resultado del coeficiente multivariado de Mardia fue 107.52, lo que indicaba falta de normalidad multivariada de los datos. Por ello, siguiendo a Finney y DiStefano (2006) se utilizó el método robusto de estimación de máxima verosimilitud (Byrne, 2001). Los índices obtenidos fueron adecuados: $\chi^{2}$ (77, $N=718)=861.48, p<.000 ; \chi^{2} /$ d.f. $=3.48 ; \mathrm{CFI}=.91 ; \mathrm{IFI}=.91 ; \mathrm{TLI}=.90 ; \mathrm{RSMR}=.05$; RMSEA = .05. Los pesos de regresión estandarizados oscilaron desde .46 a .76 .

Análisis factorial confirmatorio de la EMIPE. Con los 12 ítems de la escala del estudio 1 se llevó a cabo un análisis factorial confirmatorio con dos dimensiones. Se comprobó que los índices obtenidos no eran adecuados: $\chi^{2}(38, N=718)=525.56, p<.000 ; \chi^{2} /$ d.f. $=10.07$; $\mathrm{CFI}=.72 ; \mathrm{IFI}=.72 ; \mathrm{TLI}=.65 ; \mathrm{RSMR}=.08 ; \mathrm{RMSEA}=.11$. Se tuvo en cuenta las sugerencias mostradas por los índices de modificación y se correlacionaron los errores del ítem 1 con el ítem 2 y el ítem 11 con el ítem 12 de la dimensión de medios que exigen feedback y los errores de los ítems 4 con el 5 y el 7 con el 8 de la dimensión de medios que no exigen feedback. Se realizó un nuevo CFA y se utilizó el método de estimación de máxima verosimilitud junto con el procedimiento de bootstrapping, ya que el resultado del 
coeficiente multivariado de Mardia fue 12.79, lo que indicaba falta de normalidad multivariada de los datos. Los índices obtenidos fueron adecuados: $\chi^{2}(43, N=718)=$ $179.75, p<.000 ; \chi^{2} /$ d.f. $=3.82 ; \mathrm{CFI}=.92 ; \mathrm{IFI}=.92 ; \mathrm{TLI}=.90 ; \mathrm{RSMR}=.05 ; \mathrm{RMSEA}=$ .06. Los pesos de regresión estandarizados oscilaron desde .31 a .71 .

Análisis descriptivos y de correlaciones bivariadas

En cuanto a la escala de competencias docentes, la dimensión de competencias personales fue la más valorada seguida de las competencias específicas de Educación Física y la de competencias generales. El factor de medios de evaluación que exigen feedback presentó una mayor valoración que el de medios que no exigen feedback. Respecto al análisis de correlaciones bivariadas, todas las variables presentaron correlaciones positivas y significativas entre sí (Tabla 3).

Tabla 3. Estadísticos Descriptivos y Correlaciones de Todas las Variables

\begin{tabular}{|l|c|c|c|c|c|c|c|c|}
\hline & $M$ & $D T$ & $\alpha$ & 1 & 2 & 3 & 4 & 5 \\
\hline 1. Competencias genéricas & 2.13 & .73 & .86 & - & $.61^{* *}$ & $.61^{* *}$ & $.30^{* *}$ & $.46^{* *}$ \\
\hline 2. Competencias Educación Física & 2.47 & .58 & .86 & - & - & $.50^{*}$ & $.20^{* *}$ & $.40^{* *}$ \\
\hline 3. Competencias personales & 2.73 & .65 & .84 & - & - & - & $.19^{* *}$ & $.51^{* *}$ \\
\hline 4. Evaluación no participativa & 1.82 & .67 & .69 & - & - & - & - & $.33^{* *}$ \\
\hline 5. Evaluación participativa & 2.26 & .66 & .72 & - & - & - & - & - \\
\hline
\end{tabular}

Modelo de regresión lineal

Para comprobar el poder de predicción de las competencias docentes, se llevaron a cabo tres análisis de regresión lineal por pasos, contemplando como variable dependiente cada uno de los factores de la escala de competencias docentes y como variables predictoras las dimensiones de la escala de evaluación (Tabla 4). Respecto a la predicción sobre la dimensión competencias generales, tanto los medios que exigen feedback como los que no predijeron positiva y significativamente $(p<.001)$, con un $25 \%$ de varianza explicada. En cuanto a la dimensión competencias específicas de Educación Física, los medios de evaluación que no exigen feedback $(p<.05)$ y los que sí $(p<.001)$ predijeron de forma positiva con un $24 \%$ de varianza explicada. Se explicó un $16 \%$ de varianza sobre la dimensión competencias personales de forma positiva y significativa $(p<.001)$ a través de los medios de evaluación que exigen feedback y los que no. En todos los casos, siempre la evaluación a través de medios que exigen feedback ha presentado un mayor poder de explicación.

Análisis multivariante

Para examinar las características por sexo y grado (Primaria/CAFYD) de acuerdo a las competencias docentes y medios de evaluación, se realizaron análisis de varianza multivariados (MANOVA). Para ello, se usaron el sexo y el tipo de estudio como variables independientes, y los factores descritos anteriormente como variables dependientes. Los resultados obtenidos de los contrastes multivariados (sexo/tipo de estudios) no mostraron diferencias (Wilk's $\left.\Lambda=.99, F(5,673)=1.24, p>.05, \eta^{2}=.009\right)$. No obstante, cuando 
se analizó por separado cada grupo, se observó que por sexo se encontraron diferencias (Wilk's $\Lambda=.97, F(5,673)=2.91, p<.05, \eta^{2}=.02$ ) en la dimensión evaluación a través de medios que exigen feedback $\left(F(1,673)=2.91, p<.05, \eta^{2}=.006\right)$ a favor de las mujeres $(M=2.33, D T=.05)$ frente a los varones $(M=2.21, D T=.03)$. Por tipo de estudios también se encontraron diferencias (Wilk's $\Lambda=.95, F(5,673)=5.82, p<.001, \eta^{2}=.04$ ) en competencias generales $\left(F(1,673)=5.69, p<.01, \mathrm{\eta}^{2}=.01\right)$, competencias personales $\left(F(1,673)=1.64, p<.05, \eta^{2}=.006\right)$, medios que no exigen feedback $(F(1,673)=5.56$, $\left.p<.001, \eta^{2}=.02\right)$ y medios que sí lo exigen $\left(F(1,673)=1.99, p<.01, \eta^{2}=.007\right)$, siendo siempre mayores los valores en los estudiantes del grado de Primaria $(M=2.25, D T=.04$; $M=2.80, D T=.04 ; M=1.90, D T=.04 ; M=2.34, D T=.04$, respectivamente) frente a los de CAFYD $(M=2.03, D T=.05 ; M=2.67, D T=.04 ; M=1.68, D T=.04 ; M=2.20, D T=.04$, respectivamente).

\section{DISCUSIÓN}

El objetivo de esta investigación ha sido diseñar y validar dos instrumentos de medida, uno para medir la percepción de competencia docente y otro para medir la percepción sobre las técnicas evaluativas en estudiantes de Educación Superior de Educación Física. La hipótesis de partida se confirma, donde se ha obtenido una relación positiva entre el desarrollo de competencias docentes y los medios de evaluación utilizados, y los medios de evaluación que conllevan un feedback han predicho positivamente el desarrollo de competencias docentes en mayor medida que las técnicas no participativas.

\subsection{VALIDACIÓN DE LAS ESCALAS DE MEDIDA}

Es necesario profundizar en el análisis de las competencias adquiridas por el alumnado en la FIP para mejorar la enseñanza universitaria y el futuro laboral del estudiantado (Marzo, Pedraga \& Rivera, 2006). El diseño y validación de instrumentos de medida podrían ser una herramienta clave en el intento de optimizar el funcionamiento de la intervención docente y poder así realizar una valoración objetiva de los resultados (García \& Congosto, 2000). Con este propósito, se diseñó la escala "Percepción de la Competencia Docente en Educación Física" que presentó adecuadas medidas psicométricas. Queda compuesto por 24 ítems agrupados en tres factores que miden la percepción de competencia docente desarrollada por los estudiantes (competencia docente de Educación Física, personales y genéricas). Las competencias docentes de Educación Física hacen referencia a las específicas de la práctica docente en esta área. Las personales son aquellas indispensables para el aprendizaje de cualquier profesión. Las genéricas, las que cualquier maestro, a pesar de su especialidad, debe desarrollar.

Por otro lado, con un carácter más específico, se diseñó la "Escala de Medición de los Instrumentos y Procedimientos de Evaluación”, donde tras el análisis de investigaciones en esta línea se construyó un instrumento compuesto por 14 ítems agrupados en dos dimensiones respecto a los medios de evaluación (los que exigen feedback y los que no). Los medios que exigen feedback hacen referencia a los procesos de evaluación en los que el alumnado es participante y recibe una retroalimentación constante sobre su proceso de aprendizaje. Por el contrario, los medios que no exigen feedback son aquellos en los que 
el alumnado únicamente realiza el proceso pedido por el docente y este es el encargado de hacerle llegar una información final. También presentó unas adecuadas medidas psicométricas.

\subsection{DESARROLLO DE COMPETENCIAS GENÉRICAS, ESPECÍFICAS Y PERSONALES}

Los resultados muestran que los estudiantes perciben que desarrollan en mayor medida competencias personales, seguidas de las competencias específicas de Educación Física y, finalmente, las de competencias generales. Estos resultados coinciden con los de Arribas, Carabias y Monreal (2010), donde mostraban deficiencias en el desarrollo de algunas competencias genéricas como la implicación de las familias o la participación del docente en la vida del centro. También Gutiérrez, Pérez y Pérez (2013, p. 511), en la misma línea, concluían que existe "poco o escaso desarrollo de competencias ligadas a la gestión del centro, a la información e implicación con las familias y a la atención a la diversidad" (competencias genéricas). Camacho y Padrón (2006) también analizan las competencias desarrolladas en estudiantes de FIP y coinciden en afirmar que existen ciertas carencias sobre el desarrollo de algunas competencias genéricas, como por ejemplo el trabajo con estudiantes disruptivos, el mantenimiento de la disciplina, el desempeño de la función tutorial, el trabajo con las familias y con el equipo docente y con el control de la ansiedad, el estrés y la frustración.

También se han encontrado diferencias en la percepción de desarrollo de competencias en función del Grado que se cursa: en el Grado de Educación Primaria (especialidad de EF) se obtienen mayores valores en la percepción del desarrollo de competencias generales y personales (y, por lo tanto, perciben que las competencias específicas de Educación Física las desarrollan en menor medida). Por el contrario, en el Grado de CAFyD se obtienen mayores valores en el desarrollo de competencias específicas de Educación Física. Esto puede explicarse debido a que el modelo de formación de CAFyD históricamente está más centrado en el deporte y menos en lo pedagógico, de forma que cada vez se aleja más de lo educativo. En cambio, los estudiantes de Educación Primaria (especialidad Educación Física), tienen la visión de la necesidad de una formación inicial enfocado a los aspectos educativos. Esta idea se refuerza con la investigación de Maulini, Fraile y Cano (2015, p. 169) sobre las competencias de estudiantes de Facultades de Ciencias Motoras (en el contexto italiano), donde planteaban la necesidad de que el entrenador desarrollase competencias educativas para "no ser solamente un técnico, responsable de la preparación física y táctica de los jugadores, sino, sobre todo, un educador consciente, capaz de utilizar el deporte para transmitir valores, favorecer el aprendizaje de competencias para la vida".

\subsection{UTILIZACIÓN DE MEDIOS QUE FOMENTAN EL FEEDBACK ENTRE DOCENTE Y ESTUDIANTE}

Como se ha indicado anteriormente, el feedback y la interacción entre el docente y los estudiantes es un elemento imprescindible para que la evaluación sea formativa (Brown \& Pickford, 2013; Maclellan 2001). En investigaciones precedentes (Gutiérrez et al., 2011; Hamodi, 2014; Tabernero \& Daniel, 2012) se percibía que la utilización de exámenes era mayoritaria, y que solía ser el medio que mayor peso tenía en la calificación final. También existía cierto acuerdo en considerar que se utilizaban pocos procedimientos ligados a la evaluación formativa, como los portafolios individuales y grupales. Esto ponía de 
manifiesto que la evaluación formativa era minoritaria (Gutiérrez et al., 2013; López \& Palacios, 2012; Trillo \& Porto, 1999) por el escaso feedback que recibían los estudiantes, necesario para autorregular sus errores (fundamento de la evaluación formativa).

Pero, por el contrario, en la presente investigación los resultados muestran la percepción de los estudiantes de haber utilizado en mayor medida medios que exigen una evaluación formativa mediante la utilización del feedback (participación del estudiante en clase o en debates, portafolios, cuadernos de campo, informes o trabajos escritos, ensayos y proyectos de aprendizaje tutorados) que aquellos que no lo exigen (exámenes de preguntas abiertas, cortas, cerradas, exámenes escritos dejando disponer de documentos y exámenes orales). Esto coincide con las investigaciones más recientes (Romero et al., 2017) que ponen de manifiesto que "las prácticas de evaluación formativa y compartida están evolucionando positivamente en la universidad española" (p. 8).

Por otro lado, se ha encontrado que son las mujeres las que perciben en mayor medida que los hombres haber utilizado medios de evaluación que exigen un feedback. Esto difiere de otros trabajos donde también se han implicado a estudiantes de Educación Primaria (especialidad Educación Física) y de CAFyD, y no se han encontrado diferencias en función del sexo (Romero et al., 2017).

\subsection{RELACIÓN ENTRE MEDIOS DE EVALUACIÓN Y DESARROLLO DE COMPETENCIAS}

Cuando existe una alta percepción de desarrollo de competencias (tanto genéricas, específicas y personales), también hay alta percepción de utilización de medios de evaluación (tanto de los que exigen feedback como de los que no). Pero en todos los casos, los medios que sí exigen han presentado un mayor poder de explicación, donde todas las variables presentaron correlaciones positivas y significativas. Esto nos acerca a interpretar que la utilización de medios de evaluación más acordes con la evaluación formativa y compartida, ya que implican un feedback (como por ejemplo debates, portafolios, trabajos escritos, proyectos de aprendizaje tutorados, etc.), pueden fomentar el desarrollo de ciertas competencias docentes y personales en los estudiantes de FIP, más que los tradicionales exámenes (en cualquiera de sus versiones). Esto coincide con lo señalado por diversos autores que han puesto de manifiesto la importancia del feedback y de la interacción para el desarrollo de competencias (Biggs, 2005; Brown \& Glasner, 2003; Brown \& Pikcford, 2013; Muros \& Luis, 2012; Romero et al., 2017; Zabalza, 2007).

La investigación cuantitativa de Arribas et al. (2010) arrojaba una clara conclusión mostrando unanimidad en los tres grupos estudiados (docentes, estudiantes y egresados) sobre el convencimiento de haber logrado un mejor desarrollo de competencias profesionales cuando se utilizaron metodologías activas y sistemas de evaluación formativos. Incluso en investigaciones en las que se ha utilizado una metodología de tipo cualitativa (con grupos de discusión con estudiantes) se concluye también que los sistemas de evaluación formativa sirven para motivar el aprendizaje de los estudiantes y para que adquieran competencias profesionales (Buscà et al., 2012; Hamodi \& López, 2012).

Coincidiendo con otros autores (Barraza, 2007), se concluye, por lo tanto, que favorecer el desarrollo de competencias (genéricas, específicas y personales) no se puede lograr a través de una práctica docente tradicional, sino que se hace necesario que los formadores realicen cambios en sus procesos de enseñanza, aproximándose a sistemas de evaluación formativa y compartida. 


\subsection{LIMITACIONES Y LÍNEAS DE FUTURO}

Una de las limitaciones que presenta esta investigación es que no se trata de un estudio causal sino correlacional. Por otro lado, el estudio presenta la valoración de los estudiantes sobre su percepción de adquisición de competencias y utilización de medios de evaluación que requieren feedback o no. No obstante, hay autores (Berk, 2005) que proponen el denominado "modelo $360^{\circ}$ ", donde la retroalimentación no solo proviene de los estudiantes como en este caso, sino de múltiples fuentes. Este modelo era utilizado para la gestión y la industria desde hace medio siglo, pero según el autor es óptimo para evaluar el desempeño docente y el profesionalismo.

Como líneas de futuro se plantea la posibilidad de ampliar la muestra incluyendo a otros grados ligados a la educación (Grado en Educación Infantil, Grado en Educación Social, etc.) e incluso extrapolar la investigación a otros contextos (diferentes universidades y países) y analizar la relación entre las competencias docentes generales y personales y su relación con los medios de evaluación a través de intervenciones o estudios longitudinales.

\section{REFERENCIAS BIBLIOGRÁFICAS}

Álvarez-Méndez, J. M. (2001). Evaluar para conocer, examinar para excluir. Madrid: Morata.

ANECA (Agencia Nacional de Evaluación de la Calidad y Acreditación). (s.f.). Guía de apoyo para la redacción, puesta en práctica y evaluación de los resultados del aprendizaje. Recuperado de http://www.aneca.es/Documentos-y-publicaciones/Otras-guias-y-documentos-de-evaluacion

Arribas, J. M., Carabias, D., \& Monreal, I. (2010). La docencia universitaria en la Formación Inicial del Profesorado. El caso de la Escuela de Magisterio de Segovia. REIFOP, 13(3), 27-35.

Arribas, J. M., Manrique, J. C., \& Tabernero, B. (2014). Instrumentos de evaluación utilizados en la formación inicial del profesorado y su coherencia para el desarrollo de competencias profesionales en los estudiantes: visión del alumnado, egresados y profesorado. Revista Complutense de Educación, 27(1), 237-255

Barraza, A. (2007). La formación docente bajo una conceptualización comprehensiva y un enfoque por competencias. Estudios Pedagógicos, 33(2), 131-153.

Benito, Á., \& Cruz, A. (Eds.). (2005). Nuevas claves para la docencia universitaria en el Espacio Europeo de Educación Superior. Madrid: Narcea.

Berk, R. A. (2005). Survey of 12 Strategies to Measure Teaching Effectiveness. International Journal of Teaching and Learning in Higher Education, 17(1), 48-62.

Biggs, J. (2005). Calidad del aprendizaje universitario. Madrid. Narcea.

Boud, D., \& Falchikov, N. (2006). Aligning Assessment with Long-Term Learning. Assessment and Evaluation in Higher Education, 31(4), 399-413.

Boyle, T., \& Petriwskyj, A. (2014). Transitions to School: Reframing Professional Relationships. Early Years: An International Journal of Research and Development, 34(4), 392-404

Bretones, A. (2008). Participación del alumnado de Educación Superior en su evaluación. Revista de Educación, 347, 181-202.

Brown, S., \& Glasner, A. (Eds.). (2003). Evaluar en la Universidad. Problemas y nuevos enfoques. Madrid: Narcea.

Brown, S., \& Pickforf, R. (2013). Evaluación de habilidades y competencias en Educación Superior. Madrid: Narcea.

Buscà, F., Pintor, P., Martínez, L., \& Peire, T. (2010). Sistemas y procedimientos de Evaluación Formativa en docencia universitaria: resultados de 34 casos aplicados durante el curso académico 
2007-2008. Estudios sobre Educación, 18, 255-276.

Buscà, F., Rivera, E., \& Trigueros, C. (2012). La credibilitat dels sistemes d'avaluació formativa en la formació inicial del professorat d'educació física. Temps d'Educació, 43, 167-184.

Byrne, B. M. (2001). Structural equation modeling with AMOS: Basic concepts, applications, and programming. Mahwah, NJ: Lawrence Erlbaum Associates.

Camacho, H. M., \& Padrón, M. (2006). Malestar docente y formación inicial del profesorado: percepciones del alumnado. Revista Interuniversitaria de Formación del Profesorado, 56, 209232.

Delgado, A. M., Borge, R., García, J., Oliver, R., \& Salomón, L. (2005). Competencias y diseño de la evaluación continua y final en el Espacio Europeo de Educación Superior. Madrid: Dirección General De Universidades.

Dochy, F., Segers, M., \& Dierick, S. (2002). Nuevas vías de aprendizaje y enseñanza y sus consecuencias: Una nueva era de evaluación. Revista de Docencia Universitaria, 2(2), 13-31.

Escudero, T. (1999). Los estudiantes como evaluadores de la docencia y de los profesores: nuestra experiencia. Revista Interuniversitaria de Formación del Profesorado, 34, 69-86.

Falchikov, N. (2005). Improving Assessment Through Student Involvement. Practical solutions for aiding learning in higher and further education. Oxon: Routledge.

Finney, S. J., \& DiStefano, C. (2006). Non-normal and Categorical data in structural equation modeling. En G. Hancock \& R. O. Mueller (Eds.), Structural equation modeling: a second course (pp. 269-314). Greenwich, Connecticut: Information Age Publishing.

Gallego, J. L., \& Rodríguez, A. (2014). Percepción del alumnado universitario de Educación Física sobre su competencia comunicativa. Movimiento, 20(2), 425-444.

García, J. M., \& Congosto, E. (2000). Evaluación y Calidad del Profesorado. En T. González Ramírez (Ed.), Evaluación y Gestión de la Calidad Educativa. Un Enfoque Metodológico (pp. 127-157). Málaga: Aljibe.

Gargallo, B., Sánchez, F. J., Ros, C., \& Ferreras, A. (2010). Estilos docentes de los profesores universitarios: la percepción de los alumnos de los buenos profesores. Revista Iberoamericana de Educación, 51(4), 1681-5653.

Gessa, A. (2011). La coevaluación como metodología complementaria de la evaluación del aprendizaje: análisis y reflexión en las aulas universitarias. Revista de Educación, 354, 749-764.

González, J., \& Wagenaar, R. (2006). Tuning Educational Structures in Europe II. La contribución de las universidades al Proceso de Bolonia. Bilbao: Universidad de Deusto.

Gutiérrez, C., Pérez, A., Pérez, M., \& Palacios, A. (2011). Percepciones de profesores y alumnos sobre la enseñanza, evaluación y desarrollo de competencias en estudios universitarios de formación de profesorado. Cultura y Educación, 23(4), 499-514.

Gutiérrez, C., Pérez, A., \& Pérez, M. (2013). Percepciones de profesores, alumnos y egresados sobre los sistemas de evaluación en estudios universitarios de formación del profesorado de Educación Física. Ágora para la Educación Física y el Deporte, 15(2), 130-151.

Hamodi, C. (2014). La evaluación formativa y compartida en Educación Superior: un estudio de caso (Tesis doctoral). Universidad de Valladolid.

Hamodi, C., López, V. L., \& López, A. T. (2015a). Medios, técnicas e instrumentos de evaluación formativa y compartida en Educación Superior. Perfiles Educativos, 147(37), 146-161.

Hamodi, C., López, V. M., \& López, A. T. (2015b). Perceptions of students, graduates and teachers on learning systems evaluation. @ tic. Revista d'Innovació Educativa, 14, 71-81.

Hamodi, C., López, V. M., \& López, A. T. (2017). If I experience formative assessment whilst at University will I put it into practice later as a teacher? Formative and shared assessment in Initial Teacher Education (ITE). European Journal of Teacher Education, 40(2), 171-190.

Hamodi, C., \& López, A. (2012). La evaluación formativa y compartida en la Formación Inicial del Profesorado desde la perspectiva del alumnado y de los egresados. Psychology, Society \& Education, 4(1), 103-116. 
Hortigüela, D., Pérez-Pueyo, A., \& López-Pastor, V.M. (2015). Implicación y regulación del trabajo del alumnado en los sistemas de evaluación formativa en educación superior. Relieve: Revista Electrónica de Investigación y Evaluación Educativa, 21(1), 1-5.

Huerta, J., Pérez I. S., \& Castellanos A. R. (2000). Desarrollo curricular por competencias profesionales integradas. Revista Educar, 13, 98-104.

Ibarra, M. S., Rodríguez, G., \& Gómez, M. Á. (2012). La evaluación entre iguales: beneficios y estrategias para su práctica en la universidad. Revista de Educación, 359, 206-231.

Ivars, P., Fernández, C., \& Llinares, S. (2016). Cómo estudiantes para maestros miran de manera estructurada la enseñanza de las matemáticas al escribir narrativas. La Matemática e la Sua Didattica, 24(1-2), 79-96.

López-Pastor, V. M. (Ed.). (2006). La Evaluación en Educación Física. Revisión de los modelos tradicionales y planteamiento de una alternativa: la evaluación formativa y compartida. Buenos Aires: Miño y Dávila.

López, V. M. (2008). Desarrollando sistemas de evaluación formativa y compartida en la docencia universitaria. Análisis de resultados de su puesta en marcha en la formación inicial del profesorado. European Journal of Teacher Education, 31(3), 293-311.

López, V. M. (2011). El papel de la evaluación formativa en la evaluación por competencias: aportaciones de la Red de Evaluación Formativa y Compartida en docencia universitaria. REDU. Revista de Docencia Universitaria, 9(1), 159-173.

López, V. M., Manrique, J. C., \& Vallés, C. (2011). La evaluación y la calificación en los nuevos estudios de Grado. Especial incidencia en la Formación Inicial del Profesorado. Revista Electrónica Interuniversitaria de Formación del Profesorado, 14(4), 57-72.

López, V. M., Martínez, L. F., \& Julián, J. A. (2007). La Red de Evaluación Formativa, Docencia Universitaria y Espacio Europeo de Educación Superior (EEES). Presentación del proyecto, grado de desarrollo y primeros resultados. REDU. Revista de Docencia Universitaria, 2, 1-19.

López, V. M., \& Palacios, A. (2012). Percepción de los futuros docentes sobre los sistemas de evaluación de sus aprendizajes. Revista Teoría de la Educación: Educación y Cultura en la Sociedad de la Información, 13(3), 317-340.

Lorente, E., \& Kirk, D. (2013). Alternative democratic assessment in PETE: an action-research study exploring risks, challenges and solutions. Sport, Education and Society, 18(1), 77-96.

Maclellan, E. (2001). Assessment for learning: the differing perceptions of tutors and students. Assessment and Evaluation in Higher Education, 26(4), 307-318.

Martínez, L. F., Castejón, F. J., \& Santos, M. L. (2012). Diferentes percepciones sobre evaluación formativa entre profesorado y alumnado en formación inicial en Educación Física. REIFOP, 15(4), 57-67.

Martínez, L. F., Castejón, F. J., \& Santos, M. L. (2017). Percepciones de alumnado y profesorado en Educación Superior sobre la evaluación en formación inicial en Educación Física. Retos: Nuevas Tendencias en Educación Física, Deporte y Recreación, 32, 76-81.

Martínez, L., \& Ureña, N. (2008). Evaluación formativa y compartida en la Educación Superior: desarrollo psicomotor. Revista Española de Educación Física y Deporte, 9, 67-86.

Martínez-Mínguez, L. (2016). Proyectos de Aprendizaje Tutorados y autoevaluación de competencias profesionales en la formación inicial del profesorado. Retos: Nuevas Tendencias en Educación Física, Deporte y Recreación, 29, 242-250.

Marzo, M., Pedraga, M., \& Rivera, P. (2006). Definición y validación de las competencias de los graduados universitarios. Revista de la Educación Superior, XXXV(40), 49-70.

Maulini, C., Fraile, A., \& Cano, R. (2015). Competencias y formación universitaria del educador deportivo en Italia. Estudios Pedagógicos, 41(1), 167-182.

Moreno-Murcia, J.A., Silveira, Y., \& Belando, N. (2015). Cuestionario de evaluación de las competencias docentes en el ámbito universitario. Evaluación de las competencias docentes en la universidad. New Approaches in Educational Research, 4(1), 60-66. 
Muñoz, J.M., Ríos de Deus, M.P., \& Abalde, E. (2002). Evaluación Docente vs. Evaluación de la Calidad. Revista Electrónica de Investigación y Evaluación Educativa (RELIEVE), 8(2), 103134.

Muros, B., \& Luis, J. C. (2012). Aprendizaje, capacidades cognitivas y evaluación formativa en formación inicial del profesorado. Revista de Ciencias de la Educación, 230, 171-182.

Palacios, A., \& López, V. M. (2013). Haz lo que yo digo, pero no lo que yo hago: sistemas de evaluación del alumnado en la Formación Inicial del Profesorado. Revista de Educación, 361, 279-305.

Real Decreto 1393/2007, de 29 de octubre, por el que se establece la ordenación de las enseñanzas universitarias oficiales (BOE $\mathrm{n}^{\circ} 260$ de 30 de octubre de 2007), 44037-44048.

Romero, M. R., Castejón, F. J., López, V. M., \& Fraile, A. (2017). Formative Assessment, Communicative Competencies and ICT in Teachers Training. Comunicar, 52, 1-12.

Ruiz-Gallardo, J. R., Ruiz, E., \& Ureña, N. (2013). La evaluación en la formación inicial del profesorado: qué creemos hacer y qué perciben los alumnos. CCD. Cultura Ciencia y Deporte, $8(22), 17-29$.

Santos-Guerra, M. A. (2000). La escuela que aprende. Madrid: Morata.

Santos-Guerra, M. A. (1997). Evaluar es comprender. Buenos Aires: Magisterio del Rio de la Plata.

Semeraro, R. (2003). Università e processi di valutazione: un dibattito aperto. CADMO. Giornale Italiano di Pedagogia Sperimentale, 2, 57-79.

Tabernero, B., \& Daniel, M. J. (2012). Análisis de los sistemas de evaluación del alumnado en la formación del profesorado: estudio comparativo entre lo que opinan profesores y egresados. REIFOP (Revista Electrónica Interuniversitaria de Formación del Profesorado), 15(3), 133144.

Tejada, J. (2011). La evaluación de las competencias en contextos no formales: dispositivos e instrumentos de evaluación. Revista de Educación, 354, 731-745.

Trede, F., \& McEwen, C. (2015). Early Workplace Learning Experiences: What Are the Pedagogical Possibilities beyond Retention and Employability? Higher Education: The International Journal of Higher Education and Educational Planning, 69(1), 19-32.

Trillo, F., \& Porto, M., (1999). La percepción de los estudiantes sobre su evaluación en la Universidad. Un estudio en la Facultad de Ciencias de la Educación. Innovación Educativa, 9, 55-75.

Tuning. R. (2006). Una introducción a Tuning Educational Structures in Europe. Las contribuciones de las universidades al proceso de Bolonia. Lifelong Learning Programme. Recuperado de http://www.unideusto.org/tuningeu/images/stories/documents/General_Brochure_Spanish_ version.pdf

Vallés, C., Ureña, N., \& Ruiz, E. (2011). La Evaluación Formativa en Docencia Universitaria. Resultados globales de 41 estudios de caso. REDU. Revista de Docencia Universitaria, 9(1), 135-158.

Zabalza, M. Á. (2007). Competencias docentes del profesorado universitario: Calidad y desarrollo profesional (2a ed.). Madrid: Narcea.

Zaragoza, J., Luis, J. C., \& Manrique, J. C. (2008). Experiencias de innovación en docencia universitaria: resultados de la aplicación de sistemas de evaluación formativa. REDU. Revista de Docencia Universitaria, 4, 1-33. 
